\title{
ANNETTE Project: Contributing to The Nuclearization of Fusion
}

\author{
W. Ambrosini, L. Cizelj, P. Dieguez Porras, R. Jaspers, J. Noterdaeme, M. Scheffer, \\ and C. Schoenfelder
}

\begin{abstract}
The ANNETTE Project (Advanced Networking for Nuclear Education and Training and Transfer of Expertise) is well underway, and one of its work packages addresses the design, development and implementation of nuclear fusion training. A systematic approach is used that leads to the development of new training courses, based on identified nuclear competences needs of the work force of (future) fusion reactors and on the current availability of suitable training courses. From interaction with stakeholders involved in the ITER design and construction or the JET D-T campaign, it became clear that the lack of nuclear safety culture awareness already has an impact on current projects. Through the collaboration between the European education networks in fission (ENEN) and fusion (FuseNet) in the ANNETTE project, this project is well positioned to support the development of nuclear competences for ongoing and future fusion projects. Thereby it will make a clear contribution to the realization of fusion energy.
\end{abstract}

Index Terms - ANNETTE, competence, DEMO, education, ENEN, FuseNet, fusion, ITER, nuclear, training.

\section{INTRODUCTION}

The ANNETTE Project (Advanced Networking for Nuclear Education and Training and Transfer of Expertise, see http://www.enen-assoc.org/en/training/annette.html) is a European Horizon 2020 project that addresses the present situation of nuclear energy in Europe by a continuing effort in the field of education and training. The aim is to assure a qualified work force in the next decades by consolidating and better exploiting the achievements already reached in the past and by tackling the present challenges in preparing the European workforce in the different nuclear areas. Special attention is paid to continuous professional development, lifelong learning and cross border mobility.

Keeping in mind the attractiveness of fusion as a possible safe, sustainable and low carbon source of electricity with the potential to contribute to the future mix of different energy sources, the EU created a coherent, ambitious but pragmatic

"This work was co-funded by the European Commission under the Euratom Research and Training Programme on Nuclear Energy within the H2020 Programme, Grant Agreement Number: 661910."

W. Ambrosini is with the University of Pisa / Italy (e-mail: walter.ambrosini@ing.unipi.it).

L. Cizelj is with the Josef Stefan Institute, Ljubljana / Slovenia, (e-mail: leon.cizelj@ijs.si).

P. Dieguez Porras, is with the ENEN Association, Saclay / France (e-mail: sec.enen@cea.fr). fusion program aiming, via a comprehensive, integrated science, technology and engineering program, to provide electricity to the grid by the middle of the $21^{\text {st }}$ century. As noted in the roadmap to the realization of fusion energy (the Fusion Roadmap, see https://www.euro-fusion.org/wpcms/wpcontent/uploads/2013/01/JG12.356-web.pdf), the evolution of the fusion program requires a shift from "from pure research to designing, building and operating future facilities like ITER and DEMO. This transition requires strengthening the available engineering resources, with a marked change from non-nuclear to nuclear technologies, and has to be facilitated during Horizon 2020 by specific measures in support of training and education".

At the beginning of 2016, the ENEN (European Nuclear Education Network, see http://www.enen-assoc.org/) association together with 24 partners launched the four-year project ANNETTE. This project is co-funded by the European Commission under the Euratom Research and Training Programme on Nuclear Energy within the H2020 Programme, Call NRFP 2014-2015.

One partner of ENEN in this project is the FuseNet association (European Fusion Education Network, see http://www.fusenet.eu/), responsible for the ANNETTE Work Package 6 (WP6), dealing with Coordinating the Nuclearization of Fusion.

In its activities, FuseNet will rely on the existing initiatives and institutions in Europe that are providing education and training on nuclear (fission) related competences. In the first place, those institutions will be involved that have decided to cooperate within the ANNETTE project.

The human resources involved in development, design and construction of fusion facilities must possess suitable nuclear related competences. Through WP6 the ANNETTE project takes on this challenge. The objective is to contribute to the availability of competent human resources, as needed to enable the transition from non-nuclear to nuclear technologies described in the Fusion Roadmap. Therefore, the inclusion of

R. Jaspers is with the Eindhoven University of Technology, Eindhoven / The Netherlands (e-mail: r.j.e.jaspers@tue.nl).

J. Noterdaeme, is with the University of Ghent, Ghent / Belgium (e-mail: noterdaeme@ipp.mpg.de).

M. Scheffer is with the FuseNet Association, Eindhoven / The Netherlands (e-mail: m.scheffer@tue.nl or feo@fusenet.eu).

C. Schoenfelder is with Schoenfelder.Training, Köln / Germany (e-mail: christian@schoenfelder.training) 
WP6 within the ANNETTE project is an effective contribution to meeting one important objective of the Fusion Roadmap.

\section{THE NEED FOR NUCLEAR COMPETENCES IN FUSION}

\section{A. Why ITER is nuclear}

As already mentioned, the Fusion Roadmap stated in 2012 that the evolution of the fusion program requires "... a marked change from non-nuclear to nuclear technologies, ...".

ITER (the largest international fusion experiment), currently being built in southern France, is a nuclear facility because of the planned deuterium-tritium plasma in which the fusion reaction will be sustained through internal heating. The ITER reactor will therefore contain an inventory of radioactive material, consisting of the tritium fuel (both to start the fusion reaction and subsequently tritium is bred inside the vacuum vessel) and neutron-activated material, generated when the high-energy neutrons produced during the fusion reaction hit the wall of the reactor.

Consequently, the safety functions of ITER must ensure the confinement of radioactivity and the limitation of radiation exposure, thereby protecting the machine, the ITER staff and the environment from the radiation.

\section{B. (Nuclear) Licensing of ITER and its implications}

As a nuclear facility, the product life cycle (design, manufacturing, assembly, construction, commissioning, operation and decommissioning) of ITER must follow the applicable national (i.e. French) laws and regulations for licensing a nuclear facility. It implies that the operator (i.e. the ITER Organization / IO) is a nuclear operator that must apply for the licensing of ITER with the French nuclear safety authority (ASN, Autorité de Sûreté Nucléaire). As an example, IO had to prepare the ITER safety case, a detailed document of more than 5,000 pages, demonstrating that fusion technology is not only scientifically viable, but also safe and environmentally responsible.

However, when comparing nuclear fission with fusion reactors, the demands on licensing are less stringent. In fission, reactors must control reactivity, reactor cooling is required for a long time after reactor shutdown, the reactor core will contain a large amount of fuel (tons of Uranium), and during operation it will produce highly activated waste. In contrast, the fusion reactions will terminate intrinsically in abnormal or accidental operation scenarios. Moreover, after termination of the plasma reaction, the residual power will decrease rapidly. There is only a low quantity of fuel in the plasma, and no highly-activated waste will be produced.

In November 2012, a major licensing milestone was achieved when the French government signed a decree authorizing the creation of the ITER nuclear facility. This decree implied also that IO had to establish safety processes that must comply with French regulations. These processes must be regularly verified through audit and inspection by the French nuclear safety authority.

All this has far reaching implications. IO not only must ensure that its own activities, but also that the activities of its sub-contractors will comply with the applicable French nuclear regulation. A real challenge, as sometimes the supply chain can exist of more than 5 levels of sub-contractors.

Examples of implications on design activities include:

1. Systems and components must be safety classified, possibly also qualified for their use in ITER.

2. Relevant codes and standards (e.g. RCC-MR, Règles de conception et de construction des matériels mécaniques des installations nucléaires hautes températures, expérimentales et de fusion, see http://www.afcen.com/en/publications/rccmrx) must be applied when designing safety relevant software and equipment, e.g. Protection Important Components.

Most important is that the introduction and continuous practice of a safety culture of all personnel involved must be ensured, particularly during manufacturing, assembly, construction and commissioning activities.

Often the required competences (knowledge, skills and attitudes) will not be available with the personnel employed by the various sub-contractors of $\mathrm{IO}$, as the majority will not have experiences in nuclear (fission) projects.

\section{Relying on fission experiences}

However, these competences must be built up and should therefore exist within companies that are active in the "normal" nuclear (fission) business.

Moreover, current fission new-build projects in Europe are struggling to keep these projects within planned budget and schedule while complying with evolving safety requirements. To some degree this was caused by the insufficient competence and experience transfer between the (fission) new build period in the 1980s and the current time frame. However, by now the fission industry already has conducted appropriate activities to cope with the challenges of modern large international nuclear (new build or modernization) projects.

Consequently, resulting human resources development as well as education and training activities already pursued in the fission area could be taken as a starting point for development and implementation of necessary actions in the fusion area, and then be extended to include fusion-specific aspects and content.

\section{SEQUenCE OF WP6 ACTIVITIES}

\section{A. ANNETTE WP6 sub-tasks}

FuseNet agreed with ENEN already during preparation of the ANNETTE grant application to include the topic Nuclearization of Fusion in the ANNETTE work plan. The international cooperation within ANNETTE, and the involvement of different education and training providers specialized in nuclear issues should guarantee the success of WP6.

This work package is structured into four different sub-tasks:

1. Investigate and specify the specific competence needs for the transition of fusion to a nuclear technology

2. Design and development of fusion specific training addressing the competence needs as specified in (1),

3. Implement and evaluate courses or other forms of learning 
as designed and developed in (2),

4. Support the participation in nuclear training activities.

This approach reflects a practical implementation of the Systematic Approach to Training (SAT), which was developed in the nuclear (fission) community under the guidance of the IAEA (International Atomic Energy Agency, see [1]). SAT structures a typical training project into the main steps job and task analysis / training design / training development / training implementation / training evaluation.

\section{B. Specification of nuclear competences}

To identify and specify required nuclear competences, a workshop was organized and held in November 2016 in Eindhoven, where important fusion stakeholders were convened. Participants came from Fusion for Energy (F4E, mainly the European procurement agency for ITER), UKAEA (United Kingdom Atomic Energy Authority, operator of JET / Joint European Torus), University of Ghent, Eindhoven University of Technology, and nuclear (fission) industry, with further input from IO.

The objective was to receive input for a report on competence needs with respect to nuclearization of fusion, and to receive directions for specifying the required training.

In the report, the results had to be documented in terms of expected target groups, their required (nuclear) competences (knowledge, skills and attitudes), learning outcomes of suitable training to develop these competences, and related learning content.

\section{From specification to training development}

The list of learning outcomes and related content then was further detailed. It was transformed into a questionnaire and distributed to several European education and training institutes, preferably those that are ANNETTE partners. They were asked to provide information about their training that will develop the knowledge, skills and attitudes (i.e. learning content) as listed in the questionnaire.

This work within WP6 is currently on-going, but will be concluded in summer 2017.

Next, one will have to specify suitable criteria for prioritizing the learning content that shall be used for training development and implementation. Criteria could include the potential impact that the learning content will have on the competence development of the fusion work force.

The impact could be assessed in terms of:

- size of target group,

- the need and urgency indicated by stakeholders in the field,

- project phase in which the competences are required,

- to what extent the learning content will provide key competences that are critical for project success or performance,

- to what extent the learning content will provide competences that are indispensable because of external requirements.

- availability of existing training material within the concerned networks.

After this exercise, the fusion stakeholders will be consulted again to review and possibly verify the prioritization conducted by the WP6 team.

If training related to a specific learning content will exist, it may be directly offered and delivered to the target group, or it may have to be possibly adapted. If it does not exist, training must be newly developed. Of course, the adaptation / development actions must be possible within the scope of the ANNETTE WP6, i.e. within its budget and timing constraints.

In all cases, training development will include the activities for development of training material, and possibly also for qualification of trainers. The training material itself, and the effort for its development, will strongly depend on the training method to be applied, which must be in line with the learning outcomes that shall be achieved through the training.

In the case of knowledge competences, in most cases a faceto-face training and related slides, possibly also a training manual will be sufficient. In the case of developing skills, however, more effort must be put into development of further material, e.g. exercises, case studies, possibly also hands-on experiments or even simulations. Development of tests or examinations will further increase the development effort.

In summary, important decisions will have to be made after evaluation of the training survey, and finalization of the specification. The decisions will have an important impact on the further course of the ANNETTE WP6 project, and its relevance for the fusion stakeholders.

\section{Development and implementation of nuclear training}

Following the specification of the required training, and the selection of the target group based on a set of assessment criteria as explained in section III.C, in the upcoming phase of the ANNETTE project, (if necessary) existing training courses will be adapted, or new training courses developed. In the latter case, all different training methods will be considered for suitable application, e.g. classical presentations in classroom sessions, perhaps enhanced with exercises or case studies, elearning, hands-on or practical training, or on-the-job training.

For certain types of training, e-learning or online courses offer benefits in terms of costs and ease of access when properly designed and developed. Discussions have started with UNED (Universidad Nacional de Educación a Distancia, Spain), a FuseNet and ENEN member and project partner in ANNETTE. UNED has a broad experience and appropriate tools for implementing online courses. Consequently, pilot implementation of the first training modules may be expected soon.

The impact of pilot courses on the trainees will then be evaluated, e.g. through tests or examinations. The results and other feedback will be used to further increase the quality of the training.

\section{WP6 RESULTS So FAR}

In the project that will run until the end of 2019, FuseNet has already completed the first sub-task, and issued a report that summarizes what are the Competence Needs with Respect to Nuclearization of Fusion. A significant contribution to this report was given by fusion stakeholders through the 
stakeholders' workshop held in November 2016.

\section{A. Job positions that require nuclear competences}

One important conclusion from this workshop was that nuclear competences will be expected in a variety of different job positions that may be distinguished by

a) educational level (i.e. EQF level or craftsmen / technician / bachelor / master / PhD),

b) position within the product or system life cycle (research and development, design, manufacturing, assembly, construction, commissioning, operation, decommissioning),

c) position within an organization or a project team (e.g. engineer, senior manager, project manager),

d) position within the design, manufacturing or construction process (e.g. component or system design, design integration, chief engineer, industrial architect),

e) technical specialty (e.g. nuclear material, neutronics, electro magnetics, vacuum, tritium),

f) technical discipline (e.g. mechanical / electrical / automation engineering, physicist).

One should also consider that the individuals of the current work force active in the ongoing fusion projects (ITER, research and development for ITER and DEMO, DEMO) can be classified grossly by the two dimensions:

1. the degree to which people have previously been engaged in a nuclear (fission) environment, being either nuclear-aware, nuclearized or nuclear (see, e.g., [2], page 63),

2. the degree to which people have previously been engaged in a nuclear fusion environment, and are aware of fusion specifics (related to main components and systems of a fusion facility).

\section{B. Fusion Nuclear Engineer}

Another important statement given by fusion stakeholders was that the Fusion Nuclear Engineer must be a Nuclear Engineer (see, e.g., http://www.enenassoc.org/en/emsne/information.html) with more specialized knowledge of fusion specific topics such as vacuum technologies, cryogenic technologies, disruption phenomena, electromagnetic fields and electromagnetic loads management, and tritium waste management.

Although the problem of safety is less stringent than in a fission nuclear power plant, a Fusion Nuclear Engineer must be capable to assure the confinement of radioactive material in accidental scenarios. However, he must also assure that the machine is working in normal operation or will restart after an accidental event. These tasks require important knowledge of specialized technologies, see above. Without taking care of these fusion-specific operation aspects, a substantial risk is that the machine is so safe because it never works - but this must not happen!

Another challenge to be met will be alerting the craftsmen and technicians involved in ITER manufacturing, assembly, construction or commissioning on nuclear safety culture. The number of personnel involved, mostly from "normal" (nonnuclear) industry, will finally reach several thousands in the years to come. It will be a challenge to train them effectively on the basics of nuclear safety culture, evaluate the knowledge imparted, and document the successful evaluation.

\section{Required nuclear competences in fusion}

Based on the results of the workshop, documented in the a.m. report, the current work within WP6 focuses on specifying the required nuclear training for fusion. This will allow subsequently to identify available suitable nuclear training programs as well as existing gaps.

First, nuclear competences were derived and listed that should be considered within the scope of WP6. The competence needs in terms of Knowledge, Skills, and Attitudes were specified. It was concluded that after having attended the training (to be adapted / developed within the scope of WP6), the trainees shall have knowledge on

i. Licensing of a nuclear facility and its impact on organizational, project and personal activities,

ii. Basics of a fusion facility (i.e. its design and its operation),

iii. The multi-disciplinary nature of a fusion project, they shall be able (or have the skills) to

i. Implement nuclear regulation in their product life cycle activities,

ii. Perform the necessary processes and activities within the product life cycle,

iii. Evaluate impacts of design changes/modifications on different fields,

iv. Collaborate, communicate, and innovate within a team,

they shall exhibit a personal behavior in accordance with

i. Nuclear safety culture,

ii. Social behavior appropriate for a goal oriented team.

\section{Training specification - examples}

These training objectives (or learning outcomes) and related nuclear competences were taken as a starting point to develop the training specification. For each learning outcome, the associated learning content was identified. To achieve a better overview, the learning content was developed in a hierarchical structure in two levels, the second level providing more details. Thereby it should become easier to identify (in the next step) available training (modules).

As example, the first (knowledge oriented) training objective

Licensing and safety of a nuclear facility and its impact on organizational, project and personal activities

shall be achieved by dealing with the following Learning Content (Level one):

- Nuclear law and regulation, licensing

- Quality Management

- Codes and standards

- Risks, safety requirements, and their impact on licensing and life cycle activities

- Safety analysis and design activities

As example of a second level learning content, the last topic was broken down into the following Learning Content on Level two:

- Introduction to (deterministic and probabilistic) safety 
analysis and related tools used by different technical disciplines for simulations in support of licensing

- Development of a safety analysis report

- Thermal hydraulics: analysis by simulation, simulation tools, Computational Fluid Dynamics, risk methodologies

- AlARA (As Low As Reasonably Achievable) principles

- Radiation protection and radiation monitoring: typical simulation tools, shielding and doses etc.

\section{CONCLUSIONS}

Important stakeholders in the design, construction and operation of current and future fusion reactors, such as IO, F4E, UKAEA, and FIIF (Fusion Industry Innovation Forum, see https://www2.euro-fusion.org/fiif/), have acknowledged the need for nuclear competences. So far, WP6 in the ANNETTE project identified an excellent list of competences needs with the help of these stakeholders. This result and the overview of existing training material obtained through the survey, will provide the basis for a systematic specification of the training which needs to be adapted (when existing) or newly developed.

The collaboration between the education networks of ENEN and FuseNet, through their participation in the ANNETTE project, ensures the involvement of the fission and the fusion communities (i.e. education and training as well as research institutes plus linked industry). Through the ANNETTE project, we are therefore in a good position to contribute substantially to the realization of fusion energy, as envisioned in the Fusion Roadmap.

\section{REFERENCES}

[1] IAEA Technical Reports Series No. 380: Nuclear Power Plant Personnel Training and its Evaluation, International Atomic Energy Agency, Vienna / Austria, 1996

[2] Simonovska V., Estorff U. von: Putting into Perspective the Supply of and Demand for Nuclear Experts by 2020 within the EU-27 Nuclear Energy Sector. Report EUR 25291 EN, Petten, Netherlands, 2012 